\title{
Micromagnetic Simulation of Head-Field and Write Bubble Dynamics in Perpendicular Recording
}

\author{
Werner Scholz, Member, IEEE, and Sharat Batra, Member, IEEE \\ Seagate Research, Pittsburgh, PA 15222-4215 USA
}

\begin{abstract}
We have developed a finite-element micromagnetics model to investigate the magnetization and field dynamics of perpendicular recording heads. We have used a finite-difference micromagnetic recording model to study the influence of the head-field rise time and media velocity on the recording process. An analytical expression for the write bubble expansion velocity has been derived based on a linearized model, and we have compared it with dynamic micromagnetic simulations. It has been found to be directly proportional to the rate of change of field with time and inversely proportional to the head-field gradient. The write bubble expansion velocity needs to match or exceed the media velocity to minimize transition curvature.
\end{abstract}

Index Terms-Dynamics, micromagnetics, perpendicular recording, write bubble, write head.

\section{INTRODUCTION}

$\mathbf{T}$ HE continuous increase in areal density and data rate in magnetic recording is made possible by the reduction of the typical dimensions and time scales of various components of the recording system. However, this also requires an evermore detailed understanding of the magnetic recording media, write and read heads, electronics, and their interactions. In perpendicular magnetic recording systems, the interaction between the head and the soft underlayer (SUL), which provides a flux return path to the return pole, plays an important role since it has a strong influence on the head-field rise time [1], [2]. In this paper, we have studied the recording process on the media as a function of head field, the head-field gradient, and the head-field rise time using finite-element and finite-difference dynamic micromagnetic models.

\section{Micromagnetic Models}

We have developed a finite-element (FE) micromagnetic model to study magnetization reversal processes in write heads for perpendicular recording. The finite-element head model is based on the parallel micromagnetics code "magpar" [3], [4], which has been updated to include the magnetic field of the coils, which can be driven with an arbitrary current waveform, and a (discretized, fully micromagnetic) SUL. The head geometry is imported from a Flux3D [5] model. The current distribution in the coils and their corresponding magnetic field are calculated in Flux3D and then imported into the micromagnetic model. By simple scaling of the magnetic field of the coils, any current waveform can be simulated. The dynamic response of the recording head to the coil currents is obtained by numerical integration of the Landau-Lifshitz-Gilbert equation of motion for the magnetization using a preconditioned backward differentiation formula. In addition to the external magnetic field (of the coils), magnetocrystalline anisotropy as well as exchange and magnetostatic interactions (which include the mutual interaction between the head and the SUL) are taken

Digital Object Identifier 10.1109/TMAG.2005.855265

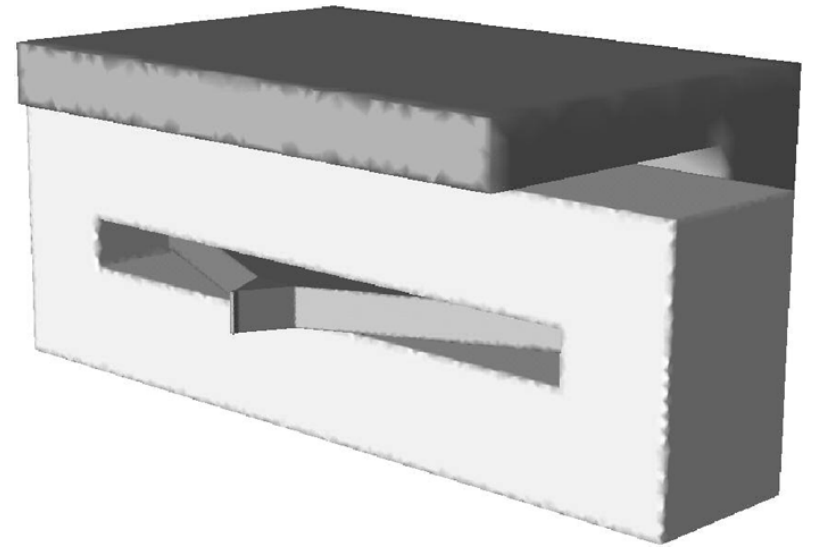

Fig. 1. Model of a single-pole-type write head for perpendicular recording. The single-turn coil is wrapped around the main pole. The magnetic circuit is closed through the SUL (not shown) to the return pole (on top).

into account. The recording layer has been omitted and eddy current or thermal effects are not taken into account.

Throughout this paper, we have used the model of a singlepole-type (SPT) write head (cf. Fig. 1) with a pole width of 40 $\mathrm{nm}$, a pole thickness of $320 \mathrm{~nm}$, and a yoke length of $3.5 \mu \mathrm{m}$ [6]. The recording head has been designed to maximize the head field and minimize the head-field rise time, but it does not address other effects, such as skew. The dynamic response of the head magnetization, which determines the head-field rise time, depends on the material properties of the head (including the damping constant), its size and shape, and the current waveform, for example, as well as the properties of the SUL. The material parameters of the pole tip have been set to a saturation magnetization of $M_{s}=1910 \mathrm{kA} / \mathrm{m}\left(J_{s}=2.4 \mathrm{~T}\right)$, an exchange stiffness of $A=13 \times 10^{-12} \mathrm{~J} / \mathrm{m}$, and an in-plane anisotropy of $K_{1}=4800 \mathrm{~J} / \mathrm{m}^{3}$, which gives an anisotropy field of $H_{K}=0.005 \mathrm{~T}$. The yoke and return pole have a saturation magnetization of $M_{s}=796 \mathrm{kA} / \mathrm{m}\left(J_{s}=1 \mathrm{~T}\right)$, an exchange stiffness of $A=13 \times 10^{-12} \mathrm{~J} / \mathrm{m}$, and an in-plane anisotropy of $K_{1}=800 \mathrm{~J} / \mathrm{m}^{3}$, while the SUL has a saturation magnetization of $M_{s}=1592 \mathrm{kA} / \mathrm{m}\left(J_{s}=2 \mathrm{~T}\right)$, an exchange stiffness of $A=10 \times 10^{-12} \mathrm{~J} / \mathrm{m}$, and an in-plane anisotropy of $K_{1}=2400 \mathrm{~J} / \mathrm{m}^{3}$. We have assumed a thickness of $300 \mathrm{~nm}$ for 
the SUL and a head-to-soft underlayer spacing of $25 \mathrm{~nm}$. The damping constant in the head and SUL has been set to $\alpha=0.2$. The head is driven by a single-turn coil, which is energized with a maximum current of $150 \mathrm{~mA}$.

In addition, we have used a micromagnetic recording model to study the write process. It uses a pseudo-Voronoi construction to generate the grain structure. The magnetic properties of the grains are defined by their saturation magnetization and average and variance of the magnetocrystalline anisotropy and intergranular exchange constants. The individual grains are modeled as Stoner-Wohlfarth particles with uniform magnetization. The magnetostatic interactions between the grains are calculated using a fast Fourier transform (FFT) technique. In this recording model, the SUL is included as a high-permeability layer, which is separated from the media layer by a nonmagnetic interlayer. It is not discretized micromagnetically. The head field is imported from a Flux3D or micromagnetic simulation as a static field map, which is suitably scaled. The recording model does not include any thermal effects.

The recording media properties were set to a media layer thickness of $12 \mathrm{~nm}$, a grain diameter of $6 \mathrm{~nm}$, a saturation magnetization of $M_{s}=579 \mathrm{kA} / \mathrm{m}$, an anisotropy field of $H_{K}=$ $1.5 \mathrm{~T}$ with a log-normal distribution and $\sigma_{H_{K}}=0.05$, and an intergranular exchange field of $0.67 \mathrm{~T}$. We assumed a head-tomedia spacing of $5 \mathrm{~nm}$ and took the head-field map at a distance of $10 \mathrm{~nm}$ from the air-bearing surface (ABS) approximately at the center of the media layer.

\section{FOOTPRINTS AND WRITE BUBBLES}

Fig. 2 shows a "footprint," which has been recorded with the write head at a fixed position and a single long current pulse on a dc-erased medium. The contour lines show the isolines for $H_{\text {eff }}=1.4$ and 1.6 T. In the following, the area within these contour lines will be referred to as the "write bubble." The effective field

$$
H_{\text {eff }}=\left(H_{\text {perp }}^{2 / 3}+H_{\text {in-plane }}^{2 / 3}\right)^{3 / 2}
$$

can be used to determine if the applied field is strong enough to switch the magnetization of a single domain particle, which follows the Stoner-Wohlfarth astroid [7]. The in-plane component of the external field reduces the energy barrier and enables switching below the anisotropy field $H_{K}=2 K_{1} / J_{s}$ as has been shown in longitudinal [8] and perpendicular [9]-[11] recording. As shown in Fig. 2, the footprint is considerably larger than the write bubble, which has to be attributed to the distribution in magnetocrystalline anisotropy of the grains, magnetostatic interactions between the grains, which (especially in the case of a dc-erased background) reduce the switching field, and intergranular exchange interactions. However, the perpendicular component of the head field is everywhere smaller than the anisotropy field of the media, which could not explain the presence of the footprint let alone its size. Thus, the effective field is the relevant field to compare the anisotropy field with.

\section{HeAd FiELd AND Write Bubble DynAmics}

After obtaining an initial magnetization distribution in zero field by static energy minimization, we excite the head with

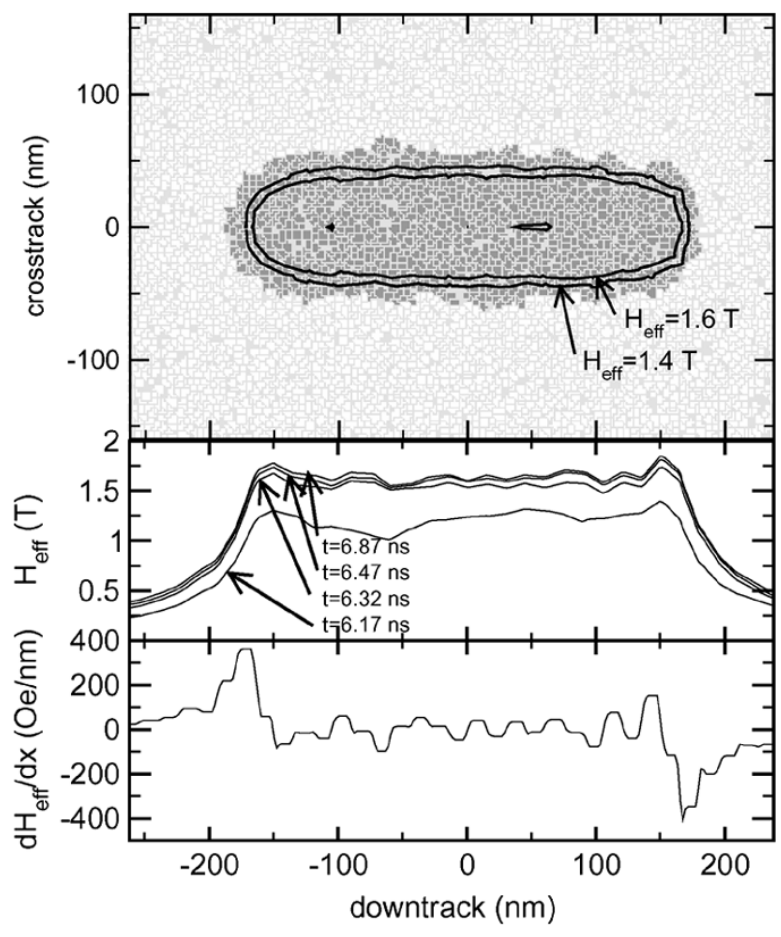

Fig. 2. Footprint and time dependence of the downtrack field profile of the effective field and field gradient of the effective field in saturation $(t>2.32 \mathrm{~ns})$.

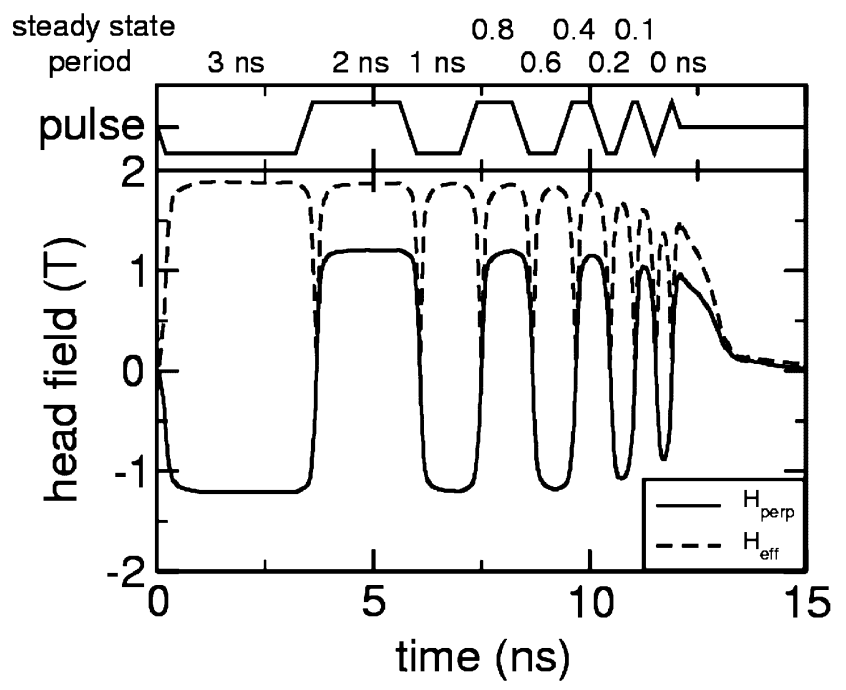

Fig. 3. Perpendicular and effective head field at the trailing edge $10 \mathrm{~nm}$ from the ABS as a function of time for current pulses with a decreasing steady-state period of constant current. The peak-to-peak current rise time is $0.4 \mathrm{~ns}$.

the magnetic field generated by the coils and integrate the Landau-Lifshitz-Gilbert equation of motion to obtain the time evolution of the magnetization. There can be a considerable phase lag between the current waveform and the head-field response [2]. We have calculated the head field as a function of time at the trailing edge of the write pole $10 \mathrm{~nm}$ from the ABS. Fig. 3 shows the results for a series of current pulses with a peak-to-peak rise time of $0.4 \mathrm{~ns}$ and increasing frequency. Below a steady-state period of $0.8 \mathrm{~ns}$, the field amplitude starts to drop, because the magnetization of the head cannot keep up with the current waveform and relax to its saturated state in the pole tip (cf. Fig. 4). Thus, the head cannot generate its 


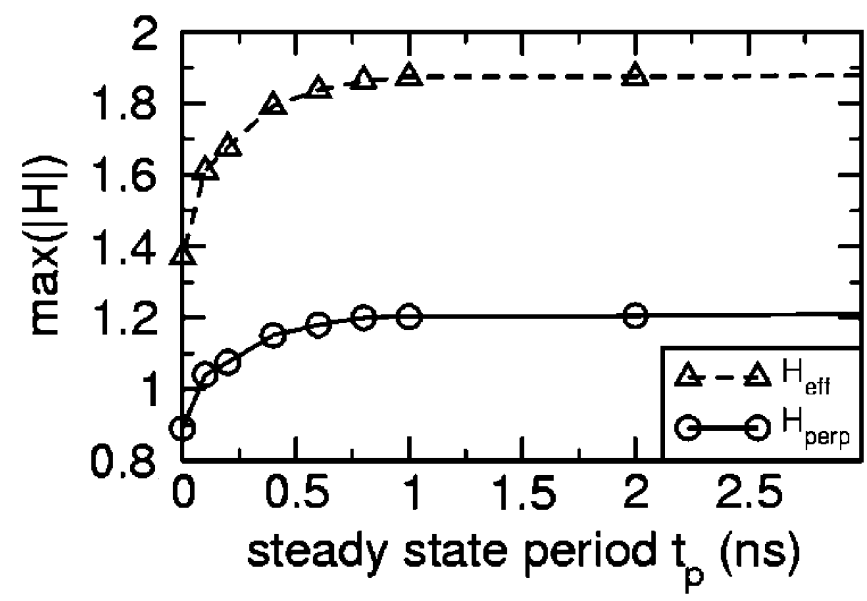

Fig. 4. Perpendicular and effective field amplitude as a function of the steadysstate period ("cell time").

maximum field, which means reduced performance. However, the performance can be improved, and the head-field rise time can be reduced by a shorter yoke, improved coil geometry, and a faster current rise time [2].

In addition to the time dependence of the head field at a single spot at the ABS (cf. Fig. 3), the micromagnetic simulation can provide the complete field map around the write pole at any time. It is very common to calculate the write field of the saturated pole using a finite-element magnetostatics program like Flux3D and scale the field map according to some assumed head-field rise time [12]. In contrast, a micromagnetic simulation provides information about the magnetic domain structure and its dynamic behavior self-consistently [13], [14]. We have checked both aspects with our time-dependent micromagnetic simulation and found that the field rise time at several spots along the pole shows a very consistent behavior. Fig. 2 illustrates this with the snapshots of the downtrack field profiles at four different points in time. The largest variations are found during reversal, when the head field is close to zero. However, this does not have an important impact on recording simulations, because then the fields are far below the switching field of the media. As the head field gets closer to its saturated state, linear scaling of the head-field profile in saturation according to the head-field rise time is a good approximation.

In order to study the dynamic recording process and the formation of transitions on the recording media, it is useful to study the "write bubble." As discussed previously, it is a reasonable approximation to use the field map of the write head in saturation and scale it according to the calculated head-field rise time function $f(t)$. A dynamic micromagnetic simulation (which includes the SUL and media layer [1]) can be used to study the writing process, but with a few approximations, we can calculate the write bubble dynamics in part analytically and compare it with the media velocity to draw some conclusions about the dynamic recording process. The write bubble expansion velocity (in downtrack direction) can be approximated analytically by linearizing the problem. Fig. 5 shows an enlarged image of the downtrack field profile $H_{\text {eff }}$ (cf. Fig. 2 at two different instants in time: $t_{0}$ and $t_{1}$. We assume that the field profile

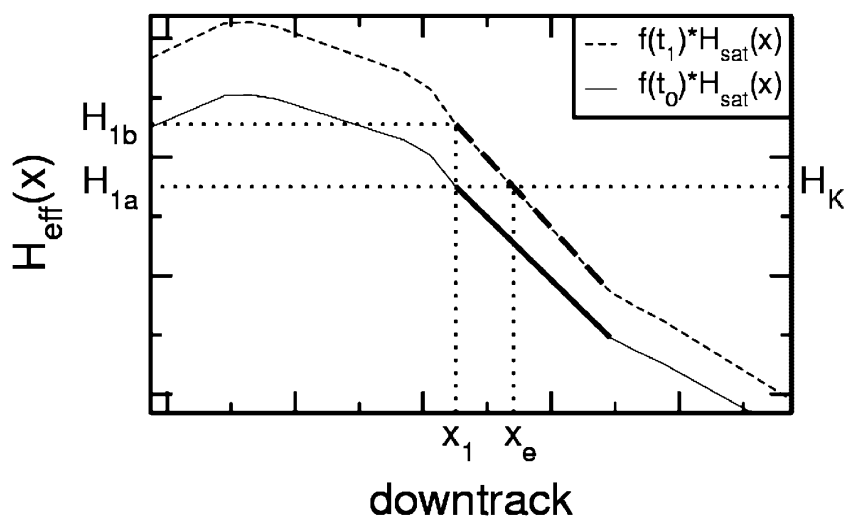

Fig. 5. Linearized model of write bubble expansion. The solid and dashed lines represent downtrack field profiles at time $t_{0}$ and $t_{1}$, respectively. The thick lines emphasize the linearized portion. At time $t_{0}$, the write bubble with $H_{\text {eff }}=H_{K}$ extends up to $x_{1}$. By time $t_{1}$, it has expanded to $x_{e}$. Thus, the write bubble velocity is given by $v_{b}=\left(x_{e}-x_{1}\right) /\left(t_{1}-t_{0}\right)$.

$H_{\text {eff }}(t, x)$ is given by simple "scaling" of the field profile in saturation $H_{\text {eff }}(t, x)=f(t) H_{\text {sat }}(x)$, where $-1 \leq f(t) \leq+1$ (although this is a good approximation only for $f(t) \approx \pm 1$ close to saturation, but this is valid when the writing takes place). Thus, the effective-field profile at time $t_{0}$ and $t_{1}$ is given by $H_{\text {eff }}\left(t_{0}, x\right)=f\left(t_{0}\right) H_{\text {sat }}(x)$ and $H_{\text {eff }}\left(t_{1}, x\right)=f\left(t_{1}\right) H_{\text {sat }}(x)$, as shown in Fig. 5.

The position of the write bubble with $H_{\text {eff }}=H_{K}=H_{1 a}$ at $t=t_{0}$ is $x_{1}$, and at $t=t_{1}$, it has expanded to $x_{e}$. Thus, the write bubble velocity is given by

$$
v_{b}=\frac{d x}{d t} \approx \frac{\Delta x}{\Delta t}=\frac{x_{e}-x_{1}}{t_{1}-t_{0}} .
$$

We can derive the write bubble velocity $v_{b}$ by writing

$$
H_{\text {eff }}(x, t)=f(t) H_{\text {sat }}(x)=H_{K} .
$$

If we take the derivative of (3) with respect to $t$, we find

$$
\frac{d f(t)}{d t} H_{\mathrm{sat}}(x)+f(t) \frac{d H_{\mathrm{sat}}(x)}{d x} \frac{d x}{d t}=0 .
$$

Solving for $v_{b}=d x / d t$ gives

$$
v_{b}(x, t)=\frac{d x}{d t}=-\frac{\frac{d f(t)}{d t} H_{\mathrm{sat}}(x)}{f(t) \frac{d H_{\mathrm{sat}}(x)}{d x}} .
$$

The negative sign stems from the fact that a field profile with negative slope gives rise to a positive bubble expansion velocity as shown in Fig. 5. In summary, the bubble velocity is proportional to the rate of change of the field with time and inversely proportional to the field gradient.

If we assume a media anisotropy field of $H_{K}=1.5 \mathrm{~T}$ and a head saturation field of $H_{\text {sat }}(x)=1.66 \mathrm{~T}$ (at the position $x$, where we want to calculate the bubble velocity for the bubble with $H_{\text {eff }}=H_{K}$ ), we find $f(t)=1.5 / 1.66=0.9$. For a rate of change of the field scaling factor $d f / d t=0.5 / \mathrm{ns}$, and a downtrack field gradient of

$$
\begin{aligned}
\frac{d H_{\mathrm{eff}}(x, t)}{d x} & =f(t) \frac{d H_{\mathrm{sat}}(x)}{d x} \\
& =0.9 \cdot(-400) \text { Oe/nm }=-360 \mathrm{Oe} / \mathrm{nm}
\end{aligned}
$$

we arrive at a bubble velocity of

$$
v_{b}=23.06 \mathrm{~m} / \mathrm{s} \text {. }
$$




\section{RECORDING}

Once the write bubble velocity has been calculated analytically as shown in the previous section or by a dynamic micromagnetic simulation, its effect on the recording process can be studied in more detail. The write bubble concept can answer the two basic questions concerning the transition location and the time, when the transition has effectively reached its final position [15], [16]. Both depend on the media anisotropy field $H_{K}$, media velocity $v_{m}$, and the bubble expansion velocity $v_{b}$.

In the following discussion, we neglect any effects of anisotropy distribution, exchange, or magnetostatic coupling between grains.

1) $v_{b}>v_{m}$ : A transition occurs as soon as the effective field exceeds the anisotropy field $\left(H_{\text {eff }}>H_{K}\right)$ approximately at the location of the write bubble with $H_{\text {eff }}=$ $H_{K}$ (cf. footprint in Fig. 2). Since the write bubble expands faster than the media velocity, the transition is continuously shifting. Its final location is reached when the bubble velocity drops below the media velocity $v_{b} \leq v_{m}$ (i.e., at $7 \mathrm{~ns}$ in Fig. 6 close to head-field saturation). The shape of the transition is determined by the shape of the write bubble at this time.

2) $v_{b}=v_{m}$ : If the write bubble velocity equals the media velocity, the transition gets written as soon as $H_{\text {eff }}>H_{K}$ (beginning of the pulse) at a location determined by the initial write bubble. As the write bubble expands and the media moves at the same speed, the transition stays at its initial location. However, since the write bubble expands also in the crosstrack direction, the transition changes its shape (curvature) as shown in Fig. 7(a) and (b). Initially, the transition shows strong curvature due to the narrow write bubble. As the bubble expands, the write bubble becomes wider, and the transition curvature is reduced.

3) $v_{b}<v_{m}$ : If the bubble velocity is slower than the media velocity, the transition gets written as soon as $H_{\text {eff }}>H_{K}$. Even though the bubble continues to expand, the transition location does not change any more, because the initial transition on the media moves away faster than the write bubble expands. This observation has an important effect on transition curvature. The initial transition is written with a very narrow write bubble with strong curvature. The write bubble continues to expand, but since the media moves away faster, the growing (and wider) write bubble cannot "reshape" the transition and reduce its curvature (as it did in the case $v_{b}=v_{m}$ ). Strong transition curvature is not desirable, because it increases the readback pulsewidth and requires a narrower reader. Narrow readers and higher readback pulsewidth reduce the signal-to-noise ratio [17], [18].

We have verified these effects using a micromagnetic recording model. Fig. 6 shows four different normalized field rise time functions $f(t)$ and their rate of change $d f(t) / d t$, which we have assumed in the different recording simulations. These field rise time profiles have been chosen to generate a single bubble velocity corresponding to the three cases discussed previously. The field map (downtrack field profile shown in Fig. 2) of the head (Fig. 1) in saturation $H_{\text {sat }}(x, y)$ has been

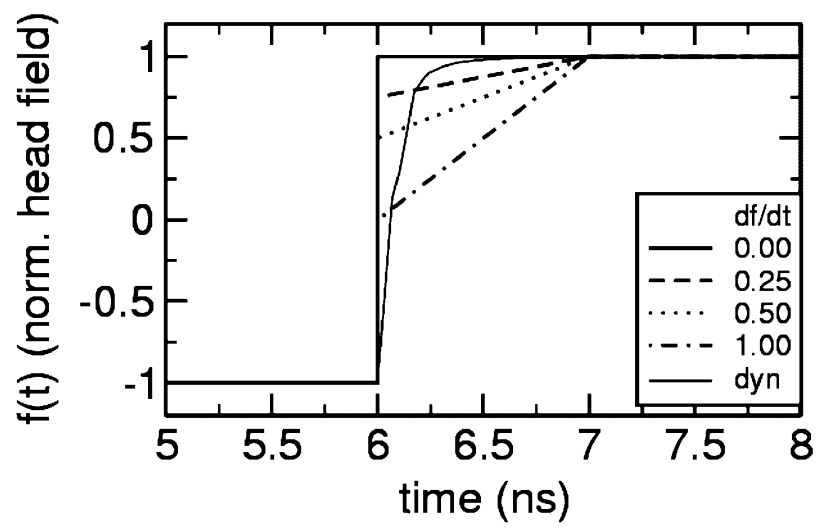

Fig. 6. Head-field rise time function $f(t)$ used for the recording simulations. The legend gives the rate of change $d f / d t$ for $6<t<7 \mathrm{~ns}$, which is proportional to the write bubble expansion velocity. "dyn" corresponds for $f(t)>0$ to the head dynamics shown in Fig. 3 .

used and scaled according to the field versus time behavior shown in Fig. 6 as $H(x, y)=f(t) H_{\text {sat }}(x, y)$. For 6 ns, we applied the full head field and wrote a mark on the ac-erased recording medium, which was moving at $25 \mathrm{~m} / \mathrm{s}$. Then, we switched the head field according to a given field versus time curve and wrote a transition as shown in Fig. 7(a) and (b). This process has been repeated 30 times on media with identical properties only with different grain and anisotropy distributions to mimic the recording at different locations on the media.

For a step function $f(t)$, the head field switches instantaneously while the recording media continues to move. Thus, the write bubble does not "expand," and $d f / d t=0$ after the switch. As a result, the shape of the transition corresponds to the shape of the footprint. However, if we assume that the head field has a finite rise time, the dynamic effects described previously come into play.

We have run micromagnetic recording simulations of all cases shown in Fig. 6 and observed the predicted behavior. In the last case (dashed-dotted line) with $d f / d t=1$, the write bubble expansion velocity is about $v_{b}=46 \mathrm{~m} / \mathrm{s}$ (if we just update $d f / d t$ in the calculation in the previous section), and thus, considerably larger than the media velocity of $v_{m}=25 \mathrm{~m} / \mathrm{s}$, which we have assumed in our simulations. As a result, we find the expected behavior: the write bubble expands continuously (after $H_{\text {eff }}>H_{K}$ ) and moves the transition until the head field saturates at $7 \mathrm{~ns}$ and the write bubble velocity drops to zero. Fig. 7(a) shows the average of 30 transitions. The transition curvature corresponds to the footprint shown in Fig. 2 because the largest write bubble effectively writes the transition as shown in Fig. 7(c).

For the intermediate case with $d f / d t=0.5$ (dotted line in Fig. 6), the write bubble velocity is $v_{b}=23 \mathrm{~m} / \mathrm{s}$, which is almost equal to the media velocity. Thus, the transition (once it is written when $H_{\text {eff }}>H_{K}$ ) remains at its initial position and changes only its shape. By the end of the simulation, its shape is comparable to that of the previous case.

The head-field rate of change $d f / d t=0.25$ gives rise to a write bubble expansion $v_{b}=11.5 \mathrm{~m} / \mathrm{s}$, which is slower than the media velocity. As discussed previously, we can expect strong transition curvature, because the expanding write bubble 


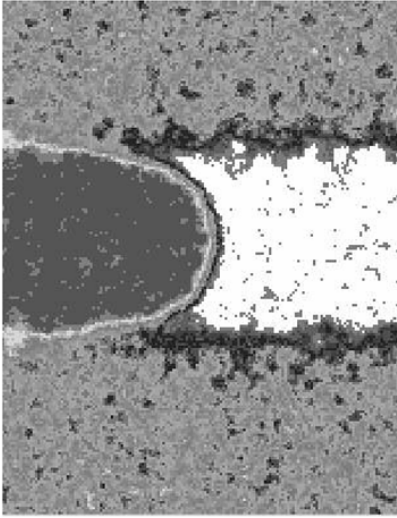

(a)

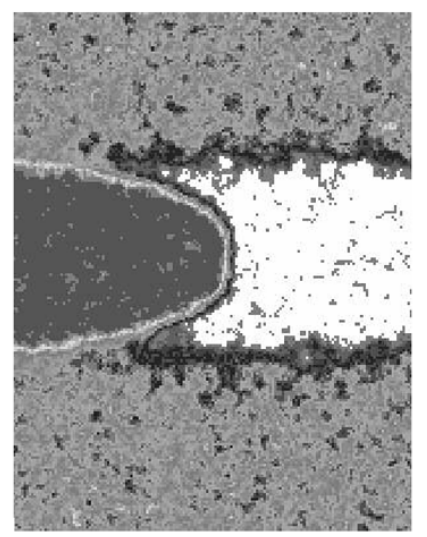

(b)

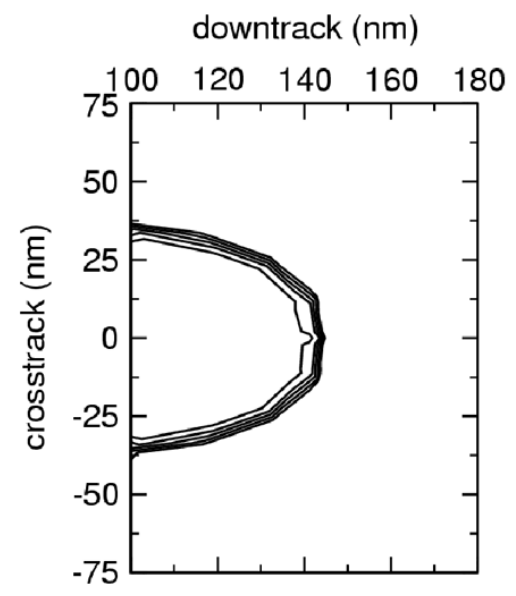

(c)

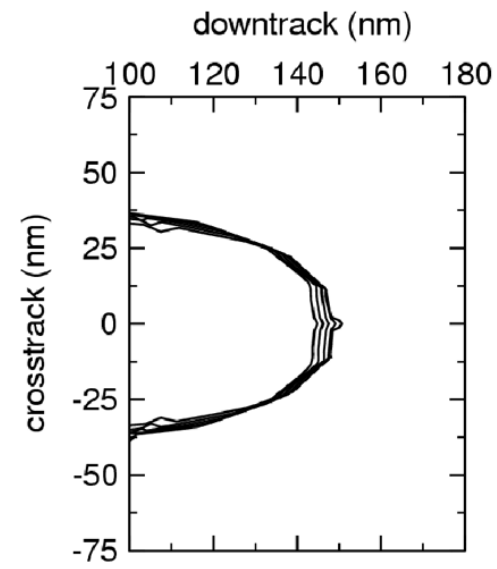

(d)

Fig. 7. Write bubble curvature for (a) fast ( $v_{b}=46 \mathrm{~m} / \mathrm{s}>v_{m}=25 \mathrm{~m} / \mathrm{s}$ ) and (b) slow ( $v_{b}=11.5 \mathrm{~m} / \mathrm{s}<v_{m}=25 \mathrm{~m} / \mathrm{s}$ ) write bubble expansion. (a) and (b) show an average of over 30 transitions on randomized grain structures. The grayscale is not proportional to the magnetization but emphasizes the transition shape. (c) and (d) show the write bubbles (enlarged for clarity) as they expand and shift corresponding to (a) and (b), respectively.

is slower than the media, and the shape of the transition is determined by the envelope of the write bubbles as the media move past the head. The micromagnetic simulation confirms this hypothesis as shown in Fig. 7(b) and (d). Similarly, an increase of the media velocity to $v_{m}=50 \mathrm{~m} / \mathrm{s}$ (while the head-field rate of change is set to $d f / d t=0.25$ ) caused strong transition curvature.

Finally, the head-field rise time function taken from the dynamic micromagnetic head simulation (Fig. 3) has been used for the recording simulations (Fig. 6). It shows a very steep rise time up to $f(t)=0.9$. Thus, the write bubble expansion velocity is still larger than the media velocity, when the write bubble has already grown very large. By the time $d f / d t$ drops below 0.5 (when the write bubble velocity gets smaller than the media velocity), the large bubble has already written a transition with low curvature comparable to the one shown in Fig. 7(a).

\section{CONCLUSION}

We have studied the head-field and write bubble dynamics of a single-pole-type write head for perpendicular magnetic recording and the effect of head-field rise time and media velocity on the recording process of isolated transitions. The
Stoner-Wohlfarth effective field, rather than the perpendicular head field alone, is the appropriate field for the recording process, when it exceeds the anisotropy field of the media. The write bubble expansion velocity of the head has been derived analytically and verified with dynamic micromagnetic simulations. It has been found to be proportional to the rate of change of the field with time and inversely proportional to the field gradient. For a fast rate of change of the head field, the write bubble velocity is greater than the media velocity and the recorded transitions show curvature, which corresponds to the footprints on static media. However, for a slow rate of change of the head field, the write bubble velocity was smaller than the media velocity and increased transition curvature has been found. The effect of the field gradient on media jitter is well known [10], [19]. However, in addition to maximizing the field gradient our study shows that the bubble velocity needs to be higher than the media velocity to minimize transition curvature.

\section{ACKNOWLEDGMENT}

The authors would like to thank G. Parker and T. Roscamp for the micromagnetic recording model and P. Asselin for review of the manuscript and helpful discussions. 


\section{REFERENCES}

[1] T. Schrefl, M. Schabes, D. Suess, F. Dorfbauer, and O. Ertl, "Fully integrated micromagnetic dynamic perpendicular magnetic recording simulations," presented at the NAPMRC 2004 Conf., Boulder, CO, Aug. 2004.

[2] W. Scholz and S. Batra, "Micromagnetic modeling of head field rise time for high data-rate recording," IEEE Trans. Magn., vol. 41, no. 2, pp. 702-706, Feb. 2005.

[3] W. Scholz, J. Fidler, T. Schrefl, D. Suess, R. Dittrich, H. Forster, and V. Tsiantos, "Scalable parallel micromagnetic solvers for magnetic nanostructures," Comput. Mater. Sci., vol. 28, pp. 366-383, 2003.

[4] Magpar-Parallel Finite Element Micromagnetics Package. [Online]. Available: http://magnet.atp.tuwien.ac.at/scholz/magpar/

[5] Flux3D. Magsoft Corp., Troy, NY. [Online]

[6] S. Batra, J. D. Hannay, H. Zhou, and J. S. Goldberg, "Investigations of perpendicular write head design for $1 \mathrm{~Tb} / \mathrm{in}^{2}$," IEEE Trans. Magn., vol. 40, no. 1, pp. 319-325, Jan. 2004.

[7] E. C. Stoner and E. P. Wohlfarth, "A mechanism of magnetic hysteresis in heterogeneous alloys," Philos. Trans. R. Soc., vol. 240, pp. 599-642, 1948.

[8] L. Zhong, Y. Zhou, J. Giusti, and J. Fernandez-de-Castro, "Head field angle-dependent writing in longitudinal recording," IEEE Trans. Magn., vol. 39, no. 3, pp. 1851-1854, May 2003.

[9] M. Mallary, A. Torabi, and M. Benakli, "One terabit per square inch perpendicular recording conceptual design," IEEE Trans. Magn., vol. 38, no. 4, pp. 1719-1724, Jul. 2002.

[10] J. S. Goldberg, P. Asselin, S. Batra, and H. Zhou, "Micromagnetic study of the correlation between head field gradient and jitter in perpendicular recording," IEEE Trans. Magn., vol. 41, no. 10, Oct. 2005.
[11] H. J. Richter and A. Yu. Dobin, "Angle effects at high-density magnetic recording," J. Magn. Magn. Mater, vol. 287, pp. 41-50, Feb. 2005.

[12] K. B. Klaassen and R. G. Hirko, "Nonlinear, eddy current damped, thin-film write head model," IEEE Trans. Magn., vol. 32, no. 5, pp. 3524-3526, Sep. 1996.

[13] D. Z. Bai and J.-G. Zhu, "A detached pole tip design of perpendicular write heads for high data-rate recording," IEEE Trans. Magn., vol. 38, no. 5, pp. 2240-2242, Sep. 2002.

[14] K. Takano, "Exact micromagnetic-FEM models of a perpendicular writer and reader," IEEE Trans. Magn., vol. 41, no. 2, pp. 696-701, Feb. 2005.

[15] R. Wood and M. Williams, "Considerations for high data rate recording with thin-film heads," IEEE Trans. Magn., vol. 26, no. 6, pp. 2954-2959, Nov. 1990.

[16] P. Thayamballi, "Modeling the effects of write field rise time on the recording properties in thin film media," IEEE Trans. Magn., vol. 32, no. 1, pp. 61-66, Jan. 1996.

[17] D. J. Seagle, M. C. Barsotti, M. L. Osborn, and V. M. Tobin, "Transition curvature analysis," IEEE Trans. Magn., vol. 35, no. 2, pp. 619-624, Mar. 1999.

[18] A. Moser, M. Xiao, P. Kappenberger, K. Takano, W. Weresin, Y. Ikeda, H. Do, and H. J. Hug, "High-resolution magnetic force microscopy study of high-density transitions in perpendicular recording media," J. Magn. Magn. Mat., vol. 287, pp. 298-302, Feb. 2005.

[19] K. Miura, H. Muraoka, and Y. Nakamura, "Effect of head field gradient on transition jitter in perpendicular magnetic recording," IEEE Trans. Magn., vol. 37, no. 4, pp. 1926-1928, Jul. 2001.

Manuscript received February 28, 2005. 